\title{
Lattice gauge-fixing for generic covariant gauges
}

\author{
L. Giusti \\ Scuola Normale Superiore, P.zza dei Cavalieri N.7 - 56100 Pisa Italy \\ INFN Sezione di Pisa, 56100 Pisa Italy \\ Dip. di Fisica, Università degli Studi di Roma "La Sapienza" \\ P.le A. Moro 2 - 00185 Rome Italy.
}

\begin{abstract}
We propose a method which allows the generalization of the Landau lattice gauge-fixing procedure to generic covariant gauges. We report preliminary numerical results showing how the procedure works for $S U(2)$ and $S U(3)$. We also report numerical results showing that the contribution of finite lattice-spacing effects and/or spurious copies are relevant in the lattice gauge-fixing procedure.
\end{abstract}




\section{Introduction}

Recently, lattice QCD Monte Carlo simulations requiring a gauge-fixing have become relevant; the gauge-fixing is essential to study gauge dependent quantities like, for example, the propagators of the fundamental fields entering the continuum QCD lagrangian and to use smearing techniques [1].

The study of gluon and quark propagators, among other things, allows a better understanding of the infrared behaviour of the theory and the confinement mechanism. Moreover, quark and gluon matrix elements can be used to obtain renormalization conditions as proposed in [2, 3].

In the last few years, numerical studies of lattice propagators have been performed by several groups: the aim of the authors [4]-[6] was to study the mechanism through which the gluon may become massive at long distances whereas more recent attempts studied its behaviour as a function of momentum [7]-[9]. Analogous studies of the quark propagator exist [6].

The existence of lattice Landau and Coulomb gauge-fixing ambiguities has been verified [10]-[13]. Studying the characteristics of these ambiguities and their influences on gauge fixed quantities is interesting at least for two reasons: the existence of these ambiguities could be the analogous of the Gribov problem in the continuum formulation of non abelian gauge theories [14] and the gauge-fixing is essential for the analytical study of the continuum limit of lattice gauge theories.

In practice, there are some cases in which it is convenient to use a gauge dependent procedure to compute gauge invariant quantities. For example, smeared fermionic interpolating operators are being used in lattice QCD spectroscopy and phenomenology. The smearing operators are gauge dependent and therefore the gauge must be fixed before they are calculated.

Up to now, the only covariant gauge for which it is known the algorithm to fix it on the lattice is the Landau Gauge [15]-[17]. This algorithm uses the original idea of Gribov [14] in the continuum, restricting the domain of integration of the partition function in the region where the functional $F(\Omega) \equiv\left\|A^{\Omega}\right\|^{2}$ reaches an extreme. Also algorithms for non covariant gauges are discussed in literature (see for example [18]).

In this paper we propose a procedure which allows to generalize the Landau gaugefixing on the lattice for a generic covariant gauge. This gauge-fixing procedure could verify the gauge independence of some results obtained in literature [4]- [9] and could allow to discriminate between gauge artifacts and true physical properties of the fundamental fields entering the QCD lagrangian. 


\section{Covariant gauges quantization}

In this section we will briefly review the general formalism to quantize a non abelian gauge theory using covariant gauge conditions [19].

Neglecting the Gribov problem, let us assume that we can find a gauge section in the space of gauge fields which intersects once and only once all gauge orbits. The Landau gauge is readily extended to an auxiliary gauge-fixing condition of the form

$$
\partial_{\mu} A_{\mu}^{\Omega}(x)=\Lambda(x)
$$

where $\Lambda(x)$ belongs to the Lie algebra of the group. Since gauge-invariant quantities should not be sensitive to changes of gauge condition, it is possible to average over $\Lambda(x)$ with a gaussian weight

$$
Z\left(J_{\mathcal{O}}\right)=\int \delta \Lambda e^{-\frac{1}{2 \alpha} \int d^{4} x T r\left(\Lambda^{2}\right)} \int \delta A_{\mu} \delta \eta \delta \bar{\eta} e^{-S(A)-S_{g h o s t}\left(\eta, \bar{\eta}, A_{\mu}\right)+\int J_{\mathcal{O}} \mathcal{O}} \delta\left(\partial_{\mu} A_{\mu}-\Lambda\right)
$$

obtaining the standard formula

$$
Z\left(J_{\mathcal{O}}\right)=\int \delta A_{\mu} \delta \eta \delta \bar{\eta} e^{-S(A)-S_{g h o s t}\left(\eta, \bar{\eta}, A_{\mu}\right)+\int J_{\mathcal{O}} \mathcal{O}} e^{-\frac{1}{2 \alpha} \int d^{4} x\left(\partial_{\mu} A_{\mu}\right)\left(\partial_{\mu} A_{\mu}\right)}
$$

where $\mathcal{O}$ is a gauge-invariant operator.

The choice $\alpha=1$ is referred to as Feynman gauge instead for $\alpha=0$ the Landau gauge is recovered.

In the next sections we will show how to implement such a formulation for a non perturbative numerical simulation to compute the mean value of a gauge-dependent observable on the lattice using the expression (2) for $Z\left(J_{\mathcal{O}}\right)$.

\section{The functional for covariant gauges}

The functional proposed by Gribov, directly in the continuum, in order to fix the Landau Gauge is

$$
F(\Omega) \equiv\left\|A^{\Omega}\right\|^{2}=\int \operatorname{Tr}\left(A_{\mu}^{\Omega}(x) A_{\mu}^{\Omega}(x)\right) d^{4} x
$$

where

$$
A_{\mu}^{\Omega}(x)=\Omega(x) A_{\mu}(x) \Omega^{\dagger}(x)-\frac{i}{g} \Omega(x) \partial_{\mu} \Omega^{\dagger}(x) .
$$

and

$$
\Omega(x)=e^{i w(x)}
$$


is a group matrix, $w(x)$ and $A_{\mu}(x)$ belonging to the Lie algebra of the group. In appendix $\mathrm{A}$ we show that

$$
\frac{\delta F(\Omega)}{\delta w^{b}}=-\frac{2}{g}\left(\partial_{\mu} A_{\mu}^{\Omega}\right)^{a} \Phi^{a b}(w)
$$

with

$$
\Phi^{a b}(w) \equiv\left[\frac{e^{\gamma}-\mathbb{I}}{\gamma}\right]^{a b} \quad \gamma^{a b} \equiv f^{a b c} w^{c}
$$

Equation (7) shows that $F(\Omega)$ is stationary when $\partial_{\mu} A_{\mu}^{\Omega}=0$.

In order to fix the gauge discussed in the section 2 we should be able to find a functional $H(\Omega)$ stationary when

$$
\partial_{\mu} A_{\mu}^{\Omega}(x)=\Lambda(x)
$$

with $\Lambda(x)$ having a gaussian distribution. The most naive way to define $H(\Omega)$ would be to find a functional $h(\Omega)$ such that

$$
\frac{\delta h}{\delta w^{b}(x)}=\frac{2}{g}\left(\Lambda^{a}(x) \Phi^{a b}(w(x))\right)
$$

so that

$$
\frac{\delta(h+F)}{\delta w^{b}(x)}=-\frac{2}{g}\left(\partial_{\mu} A_{\mu}^{\Omega}(x)-\Lambda(x)\right)^{a} \Phi^{a b}(w(x)) .
$$

However we will now show that this is not possible; in fact for a non abelian gauge theory does not exist a functional satisfying (10). A necessary condition for the existence of such a functional would be

$$
\frac{\delta^{2} H(\Omega)}{\delta w^{c}(x) \delta w^{b}(y)}=\frac{\delta^{2} H(\Omega)}{\delta w^{b}(y) \delta w^{c}(x)}
$$

which implies the integrability condition

$$
\frac{\delta}{\delta w^{c}(x)}\left(\Lambda^{a}(y) \Phi^{a b}(w)\right)=\frac{\delta}{\delta w^{b}(y)}\left(\Lambda^{a}(x) \Phi^{a c}(w)\right) .
$$

Expanding $\Phi^{a b}(w(x))$ in power of $w(x)$, equation (13) should be satisfied order by order in $w(x)$. From equation (8) we have

$$
\Phi^{a b}(w) \simeq \delta^{a b}+\frac{\gamma}{2}^{a b}=\delta^{a b}+f^{a b c} \frac{w^{c}}{2},
$$


Equation (13) is then in contrast with the antisymmetry of $f^{a b c}$.

The functional we propose in order to fix the gauge discussed in section 2 is:

$$
H(\Omega)=\int d^{4} x \operatorname{Tr}\left[\left(\partial_{\mu} A_{\mu}^{\Omega}-\Lambda\right)\left(\partial_{\nu} A_{\nu}^{\Omega}-\Lambda\right)\right] .
$$

In fact

$$
\frac{\delta H(\Omega)}{\delta w^{a}}=2 \Phi^{a b}(w)\left[D_{\nu} \partial_{\nu}\left(\partial_{\mu} A_{\mu}^{\Omega}-\Lambda\right)\right]^{b}
$$

which shows that $H(\Omega)$ is stationary when

$$
\partial_{\mu} A_{\mu}^{\Omega}(x)-\Lambda(x)=0
$$

The problem is to show that no spurious stationary points of (15) exist. In fact, for example, apparently

$$
\partial_{\mu} A_{\mu}^{\Omega}(x)-\Lambda(x)=\text { cost } .
$$

also satisfies

$$
\frac{\delta H(\Omega)}{\delta w^{a}}=0
$$

and other non trivial solutions of the equation (19) could exist as well. In our case we can exclude the possibility of constant zero modes. In fact integrating the equation (18) we obtain

$$
\int \partial_{\mu} A_{\mu}^{\Omega}(x) d^{4} x-\int \Lambda(x) d^{4} x=\operatorname{cost} \int d^{4} x .
$$

Through the use of periodic boundary conditions we have

$$
-\int \Lambda(x) d^{4} x=\operatorname{cost} \int d^{4} x .
$$

Equation (20) assures that cost $=0$ when $\Lambda(x)$ has a gaussian distribution. This result assures that the functional $H(\Omega)$ does not have spurious stationary points satisfying equation (18).

It is interesting to note that for $\Lambda(x)=0$ the functional $H(\Omega)$ has absolute minima for any $\Omega$ satisfying $\partial_{\mu} A_{\mu}^{\Omega}(x)=0$. This is not the case for the Gribov functional.

\section{Covariant gauges on the Lattice}

The gauge variables of a compact lattice gauge theory are the links $U_{\mu}(x)$ and they are elements of the gauge group.

In this section we outline a procedure to compute numerically the mean value 
of a gauge dependent operator $\mathcal{O}$ on the lattice using a generic covariant gauge quantization. The mean value of a gauge dependent operator on the lattice is defined as :

$$
\langle\mathcal{O}\rangle=\int \delta \Lambda e^{-\frac{1}{2 \alpha} \int d^{4} x \operatorname{Tr}\left(\Lambda^{2}\right)} \int d U \Delta_{f}(U) \delta(\Delta-\Lambda) e^{-S_{W}(U)} \mathcal{O}(U) .
$$

where $S_{W}(U)$ is the Wilson lattice gauge invariant action, $\Delta_{f}(U)$ is the FaddeevPopov determinant and

$$
\Delta(x)=\frac{1}{2 i a g} \sum_{\mu=1}^{4}\left[U_{\mu}(x)-U_{\mu}^{\dagger}(x)\right]_{\text {traceless }}-\left[U_{\mu}(x-\mu)-U_{\mu}^{\dagger}(x-\mu)\right]_{\text {traceless }} .
$$

The computation of the integral (21) can be schematized as follows:

- A gauge configuration $\{U\}$ with periodic boundary conditions according to the gauge invariant weight $e^{-S_{W}(U)}$ is generated;

- For each $\{U\}$ configuration random matrices $\Lambda(x)$ belonging to the group algebra are extracted according to the weight $e^{-\frac{1}{2 \alpha} \operatorname{Tr} \Lambda^{2}(x)}$;

- Given $\Lambda(x)$, a numerical algorithm extremizes a discretization of the functional $H(\Omega)$. This defines the lattice gauge-fixing condition

$$
\Delta(x)-\Lambda(x)=0 \quad \forall x ;
$$

- the mean value of the lattice gauge dependent operator is then defined as

$$
\langle\mathcal{O}\rangle^{\text {Latt }}=\frac{1}{N} \sum_{\{\operatorname{con} f\}} \mathcal{O}\left(U_{i}\right)
$$

Since $\Lambda(x)$ has a gaussian distribution for all $x$, if the number of lattice sites is sufficiently large

$$
\frac{1}{V} \sum_{x} \Lambda(x)=\bar{\Lambda}=0 .
$$

\section{$5 \quad$ Lattice gauge-fixing for covariant gauges}

A possible discretization of $H(\Omega)$ is

$$
H_{L}(\Omega)=\frac{1}{V} \operatorname{Tr} \sum_{x}\left[\Delta^{\Omega}(x)-\Lambda(x)\right]^{2}
$$

where

$$
\Delta^{\Omega}(x)=\frac{1}{2 i a g} \sum_{\mu=1}^{4}\left[U_{\mu}^{\Omega}(x)-U_{\mu}^{\Omega \dagger}(x)\right]_{\text {traceless }}-\left[U_{\mu}^{\Omega}(x-\mu)-U_{\mu}^{\Omega \dagger}(x-\mu)\right]_{\text {traceless }},
$$


$\Lambda(x)$ are matrices which belong to the group algebra, $V$ is the lattice volume and links transform under a gauge transformation as

$$
U_{\mu}^{\Omega}(x)=\Omega(x) U_{\mu}(x) \Omega^{\dagger}(x+\mu) .
$$

$H_{L}(\Omega)$ is the simplest discretization of $H(\Omega)$. For a generic transformation close to $\Omega^{0}$ we define

$$
\left.\frac{\delta H}{\delta \Omega}\right|_{\Omega^{0}} \equiv \frac{\delta H}{\delta \epsilon} \quad, \quad \Omega(x)=\Omega^{0}(x)(\mathbb{I}-i \epsilon(x))
$$

and we have

$$
\begin{array}{r}
\frac{\delta H_{L}}{\delta \epsilon^{a}}=-\frac{1}{V g a} \sum_{\mu} \operatorname{Tr}\left[T ^ { a } \left(( \Delta ^ { \Omega ^ { 0 } } ( x ) - \Lambda ( x ) ) \left(U_{\mu}^{\Omega^{0} \dagger}(x)+\right.\right.\right. \\
\left.U_{\mu}^{\Omega^{0}}(x)+U_{\mu}^{\Omega^{0} \dagger}(x-\mu)+U_{\mu}^{\Omega^{0}}(x-\mu)\right)+ \\
-\left(\Delta^{\Omega^{0}}(x-\mu)-\Lambda(x-\mu)\right)\left(U_{\mu}^{\Omega^{0} \dagger}(x-\mu)+U_{\mu}^{\Omega^{0}}(x-\mu)\right)+ \\
\left.\left.\left(\Delta^{\Omega^{0}}(x+\mu)-\Lambda(x+\mu)\right)\left(U_{\mu}^{\Omega^{0} \dagger}(x)+U_{\mu}^{\Omega^{0}}(x)\right)\right)\right]
\end{array}
$$

where $T^{a}$ are the group generators.

We have to find a gauge transformation $\Omega$ such that $\left\{U_{\mu}^{\Omega}\right\}$ satisfies

$$
\frac{\delta H_{L}}{\delta \epsilon^{a}(x)}=0 \forall x
$$

In order to solve numerically equation (28) we use an iterative algorithm for the minimization of $H_{L}(\Omega)$. If such an algorithm converges (this is not guaranteed and must be checked in practice), its fixed points will be configurations satisfying the condition (28).

In order to study the convergence of the algorithm, two lattice quantities can be monitored

$$
\begin{aligned}
H_{L} & =\frac{1}{V} \operatorname{Tr} \sum_{x}[\Delta(x)-\Lambda(x)]^{2} \\
\vartheta_{H} & \equiv V \sum_{x, a} \frac{\delta H_{L}}{\delta \epsilon^{a}} \frac{\delta H_{L}}{\delta \epsilon^{a}} .
\end{aligned}
$$

The function $H_{L}(\Omega)$ is defined on a compact set. If the numerical algorithm of minimization converges, $\vartheta_{H}$ must go to a value of the order of the precision required for the minimization whatever starting configuration we use. The procedure works if also $H_{L}$ goes to a value of the order of the precision required for the minimization 
for those configurations where the algorithm converges. In the next section we will show numerical results suggesting that $H_{L}(\Omega)$ goes to zero when it is minimized for the configurations considered.

We remark that the same discretized definition of gauge field $(1 / 2 i a g)\left[U_{\mu}(x)-\right.$ $\left.U_{\mu}^{\dagger}(x)\right]_{\text {traceless }}$ is used in the definition of $H_{L}(\Omega)$ and of $\Delta^{\Omega}(x)$.

On the contrary in the Landau lattice gauge-fixing procedure, usually adopted in the literature, different definitions of the lattice gauge potential are used. In section 7 we report numerical results showing interesting phenomena arising if different lattice gauge potential definitions are used in the lattice Landau gaugefixing procedure.

\section{Numerical Simulations}

In this section we report preliminary results of some numerical simulations. In this exploratory study we considered $S U(2)$ and $S U(3)$ non thermalized configurations with links defined as

$$
U_{\mu}(x)=e^{i b B_{\mu}\left(x+\frac{\mu}{2}\right)}
$$

with

$$
B_{\mu}\left(x+\frac{\mu}{2}\right)=\sum_{a} B_{\mu}^{a}\left(x+\frac{\mu}{2}\right) T^{a}
$$

where $B_{\mu}^{a}\left(x+\frac{\mu}{2}\right)$ are smooth functions of $x$ (typically $\sin (x)$ and $\left.\cos (x)\right), T^{a}$ are group generators and " $b$ " is a parameter which determines the distance of $U_{\mu}(x)$ from identity. We generated, on a volume $V=4^{4}$, nine configurations for each group, three for each value $b=0.1, b=0.3, b=0.7$. Since the function $H_{L}(\Omega)$ is defined on a compact set, the procedure works whatever configuration we start from, except for numerical convergence problems. A complete study of this numerical algorithm for thermalized Monte Carlo configurations will be presented in a future paper.

The gauge-fixing algorithm implements an iterative minimization for $H_{L}(\Omega)$ which updates link matrices via $S U(2)$ subgroups, as proposed in ref. 20]. We monitored the quantities $H_{L}$ and $\vartheta_{H}$ after every lattice sweep of the gauge-fixing algorithm. We mention that after each gauge-fixing sweep we orthogonalized the link variables, to make sure that they have not been driven off the gauge group.

In fig. 1 we plot $H_{L}$ and $\vartheta_{H}$ as a function of the number of gauge-fixing sweeps for a configuration of $S U(3)$ with $a=0.3$ and $\Lambda(x)=0$. It is easy to see that both $H_{L}$ and $\vartheta_{H}$ go to values consistent with the precision required for gaugefixing $\left(\leq 10^{-5}\right)$. For all configurations we generated the behaviour of $H_{L}$ and $\vartheta_{H}$ is consistent with that shown in fig.1. For $\Lambda(x)=0$ we fixed the gauge condition 


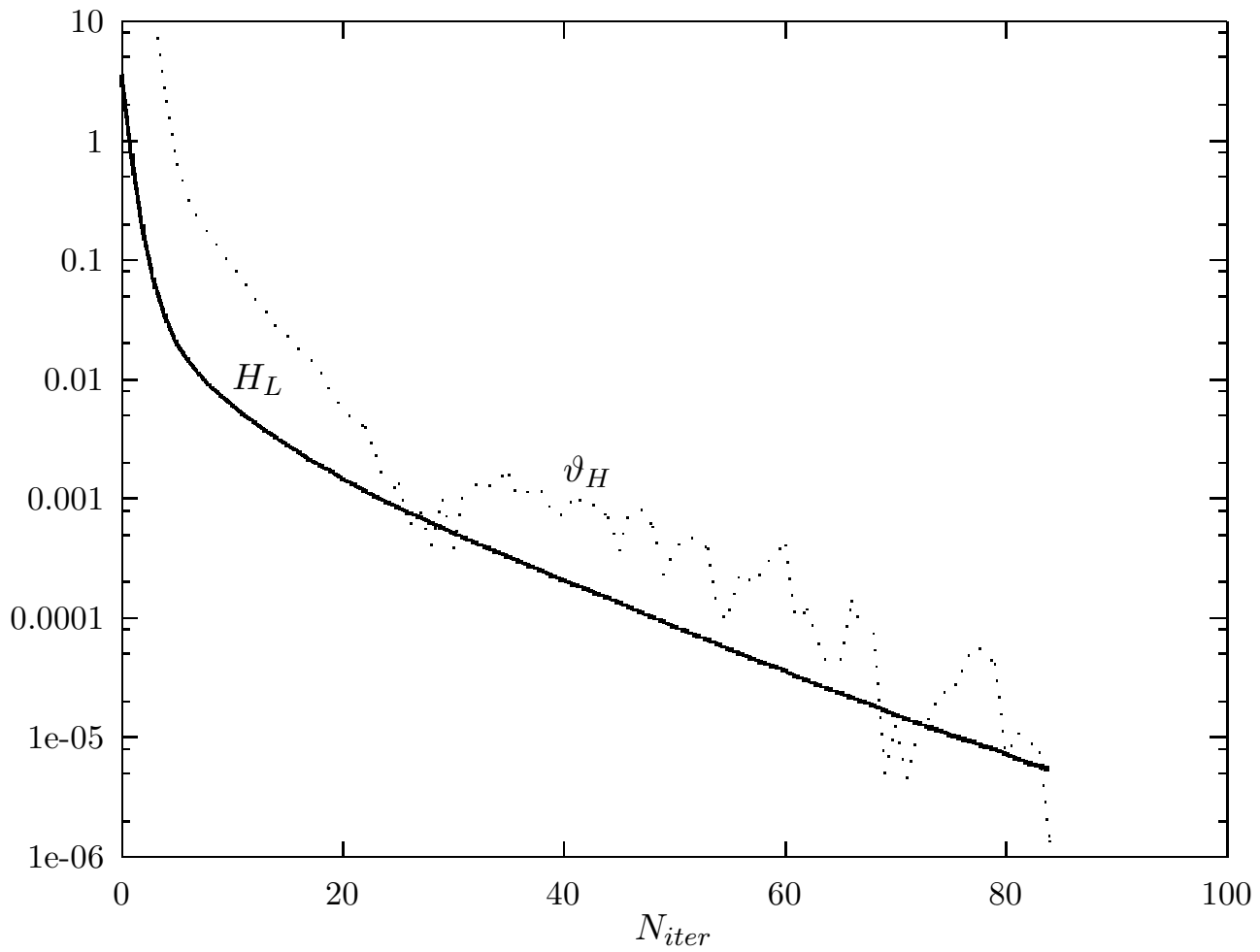

Figure 1: Values of $H_{L}$ and $\vartheta_{H}$ as functions of number of iterations $N_{\text {iter }}$ of the covariant gauge-fixing algorithm obtained extremizing $H_{L}$ for a configuration of $S U(3)$ with $a=$ $0.3, V=4^{4}$ and $\Lambda(x)=0$.

$\Delta(x) \leq 10^{-5}$ for each configuration also using the old Landau gauge-fixing algorithm. For the two configurations obtained with the two algorithms, we measured the discretization of $F(\Omega)$

$$
F_{L}(\Omega)=-\frac{1}{V} \sum_{x, \mu} \operatorname{Re} \operatorname{Tr} U_{\mu}^{\Omega}(x)
$$

as a measure of the distance between the two configurations. The values are the same with a precision of $10^{-5}$, consistent with the precision required to minimize $H_{L}$.

In fig. 2 we plot $H_{L}$ as a function of the number of gauge-fixing sweeps for a configuration of $S U(2)$ with $a=0.1$ and $\Lambda(x) \neq 0$. 


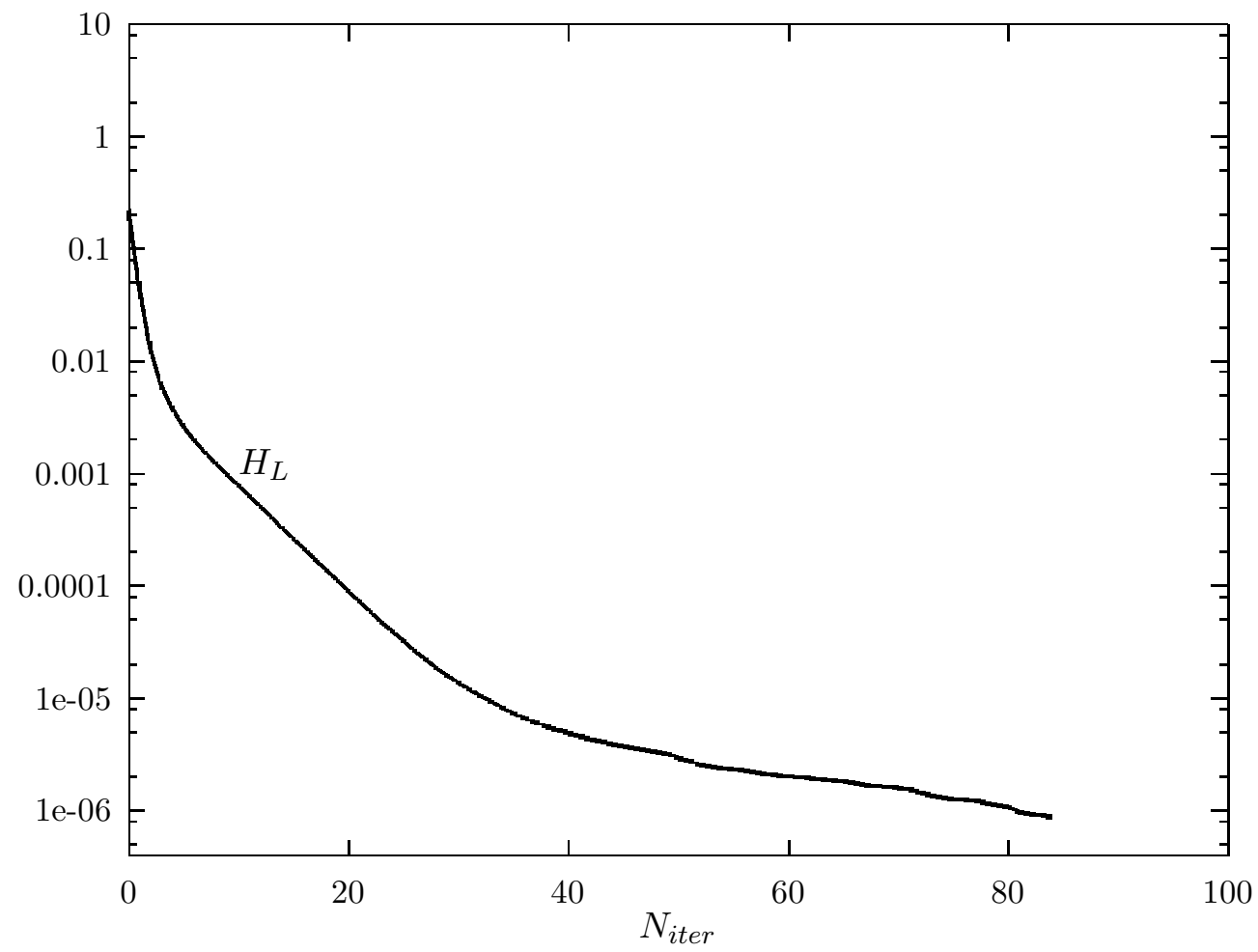

Figure 2: Values of $H_{L}$ and $\vartheta_{H}$ as functions of number of iterations $N_{\text {iter }}$ of the covariant gauge-fixing algorithm obtained extremizing $H_{L}$ for a configuration of $S U(2)$ with $a=$ $0.1, V=4^{4}$ and $\Lambda(x) \neq 0$.

In fig. 2 it is easy to see that the behaviour of the algorithm when $\Lambda(x) \neq 0$ is similar to that one with $\Lambda(x)=0$.

Finally we stress that the numerical minimization of the functional (25) is more complicated than that of $F_{L}(\Omega)$. In this exploratory study the time per iteration required to minimize $H_{L}(\Omega)$ is $5-10$ times the time per iteration required to minimize $F_{L}(\Omega)$. 


\section{Comments on the lattice Landau gauge-fixing}

In this section we report some numerical results which show some consequences of using different lattice definition of gauge potential in the Landau lattice gaugefixing procedure.

The standard way of fixing the Landau gauge on the lattice is based on the minimization of the function $F_{L}(\Omega)$ defined in the equation (29) which is a particular discretization of $F(\Omega)$ with

$$
\left[A_{\mu}^{2}\right]^{L a t}=-\frac{1}{g^{2} a^{2}}\left(U_{\mu}+U_{\mu}^{\dagger}-2 \mathbb{I}\right) .
$$

Once $F_{L}(\Omega)$ has been numerically minimized, the quantity

$$
\vartheta_{F}=\frac{1}{V} \sum_{x} \operatorname{Tr}\left[\Delta(x) \Delta^{\dagger}(x)\right]
$$

is supposed to vanish and this is the signal that the lattice gauge condition $\Delta(x)=0$ has been achieved at each site. $\Delta(x)$ is a discretization of $\partial_{\mu} A_{\mu}(x)$ with

$$
\left[A_{\mu}\right]^{\text {Lat }}=\frac{\left[U_{\mu}(x)-U_{\mu}^{\dagger}(x)\right]_{\text {traceless }}}{2 \text { iag }} .
$$

Some authors showed that, in the lattice Landau Gauge, lattice copies characterized by different values of $F_{L}$ exist. These solutions of $\Delta^{\Omega}(x)=0$ are indeed different gauge-related configurations on the lattice not connected by a colour rotation. However it is important to note that the definition of the lattice gauge potential used in the discretization of $F(\Omega)$ does not correspond to the definition used in the discretization of the divergence $\Delta(x)$. This implies that two different lattice definitions of $A_{\mu}$ have been used to check the Landau gauge-fixing condition and to tag two different solutions.

The authors in ref. 223, 24 have found two ensembles of "lattice copies" with $V=$ $16^{3} \times 32$ and $\beta=6.0$. To analyse these configurations we used the usual $F_{L}$ and $\vartheta_{F}$ variables defined in equations (29,31) and another function defined as

$$
\vartheta_{F}^{\prime}=\frac{1}{V} \sum_{x} \operatorname{Tr}\left[\Delta^{\prime}(x) \Delta^{\prime \dagger}(x)\right]
$$

where

$$
\begin{aligned}
\Delta^{\prime}(x) & =\frac{1}{4 i a g} \sum_{\mu=1}^{4}\left[\left(U_{\mu}(x)-U_{\mu}^{\dagger}(x)\right)\left(U_{\mu}(x)+U_{\mu}^{\dagger}(x)\right)\right]_{\text {traceless }} \\
& -\left[\left(U_{\mu}(x-\mu)-U_{\mu}^{\dagger}(x-\mu)\right)\left(U_{\mu}(x-\mu)+U_{\mu}^{\dagger}(x-\mu)\right)\right]_{\text {traceless }}
\end{aligned}
$$


We stress that $\Delta$ and $\Delta^{\prime}$ are two discretized definitions of $\partial_{\mu} A_{\mu}$ which tend to the same expression as $a \rightarrow 0$. For each "lattice copy" we measured the values of $F_{L}$, $\vartheta_{F}$ and $\vartheta_{F}^{\prime}$ which we report on table 1. These numerical results show that the

\begin{tabular}{|c|c|c|c|c|}
\hline Ensemble & Copy & $F_{L}(\Omega)$ & $\vartheta_{F}$ & $\vartheta_{F}^{\prime}$ \\
\hline $\mathrm{A}$ & 1 & 2.583760909874 & $2.006239333971 E-10$ & 0.4114643051772 \\
& 2 & 2.584445214939 & $3.0326250142058 E-10$ & 0.4090001249674 \\
& 3 & 2.584426547617 & $8.4843522906603 E-10$ & 0.4091814689158 \\
& 4 & 2.583991349769 & $8.2208712589082 E-10$ & 0.4106076010266 \\
& 5 & 2.584036530091 & $2.8552454724294 E-10$ & 0.4097852320118 \\
& 6 & 2.58354405348 & $3.3687550405651 E-10$ & 0.4117689556597 \\
\hline $\mathrm{B}$ & 1 & 2.581884641098 & $2.5513621387496 E-10$ & 0.4177011836703 \\
& 2 & 2.582231848321 & $7.5376561548352 E-10$ & 0.4159162059697 \\
& 3 & 2.58231860688 & $6.1085384335158 E-10$ & 0.4159582027108 \\
\hline
\end{tabular}

Table 1: Final values of $\vartheta_{F}$ and $\vartheta_{F}^{\prime}$ after gauge-fixing algorithm which extremizes numerically the functional $F_{L}$ for two gauge fixed ensemble of configuration with $V=16^{3} \times 32$ and $\beta=6.0$.

finite lattice spacing effects and/or spurious copy contributions to $\vartheta_{F}$ and $\vartheta_{F}^{\prime}$ are of the order of $10^{-1}$ while the difference between the values of $F_{L}$ for two different copy are of order $10^{-3}$ when $\vartheta_{F} \leq 10^{-10}$. This shows that with this method it is not possible to decide if two different solutions of Landau lattice gauge-fixing condition correspond to different Gribov copies in the continuum. This procedure is not apt to decide if the lattice multiple solution problem of $\Delta^{\Omega}(x)=0$ has an analogy with the continuum Gribov problem.

Moreover we observe that for a lattice copy there is a big difference between the values of the two discretizations $\vartheta_{F}$ and $\vartheta_{F}^{\prime}$ respect to the precision required for the minimization. It would be interesting to understand if the difference between $\vartheta_{F}$ and $\vartheta_{F}^{\prime}$ is only due to the higher order lattice spacing effects or to spurious copies 25]. The problem of higher order contributions and/or spurious solutions to $\vartheta_{F}$ afflicts all numerical computations of gauge dependent operators. In matching numerical results obtained on the lattice with the corresponding continuum formulas one must carefully evaluate the error assigned to the gauge-fixing condition even if $\vartheta \leq 10^{-10}$. Moreover the residual gauge freedom associated to lattice copies tagged with the functional (29) should not induce effects higher than the sistematic uncertainty due to the higher order contributions and/or spurious solutions. 
The numerical results shown in this section indicate also the importance to improve the gauge-fixing algorithm on the lattice also for the Landau gauge.

\section{Conclusions}

We have proposed a method which allows the generalization of the Landau lattice gauge-fixing procedure to generic covariant gauges. We have shown that a functional whose stationary points are $\partial_{\mu} A_{\mu}^{\Omega}(x)=\Lambda(x)$ cannot be obtained as a direct generalization of the Landau lattice gauge-fixing functional used in literature. In the continuum we proposed a functional reaching an extreme when $\partial_{\mu} A_{\mu}^{\Omega}(x)=\Lambda(x)$ and we used the simplest discretization of $H(\Omega)$ to fix numerically a generic covariant gauge on the lattice. We reported preliminary numerical results showing how this procedure works for $S U(2)$ and $S U(3)$. Numerical results also show that the contribution of finite lattice-spacing effects and/or spurious copies are relevant in the lattice gauge-fixing procedure and must be carefully evaluate.

\section{Acknowledgement}

It's a great pleasure to thank M. Testa for having suggested and supervised this work. I warmly thank G. Martinelli, S. Petrarca, M. Testa and A. Vladikas for many useful discussions, suggestions and encouragements throughout this work.

\section{Appendix A}

In this appendix we calculate the variation of a $S U(N)$ matrix

$$
\begin{aligned}
\Omega & =e^{i w} \\
w & =\sum_{a} w^{a} T^{a}
\end{aligned}
$$

and of the functional

$$
F(\Omega)=\int d^{4} x \operatorname{Tr}\left(A_{\mu}^{\Omega}(x) A_{\mu}^{\Omega}(x)\right)
$$

for an infinitesimal variation of $w$ [21]. Following Feynman [22], $\Omega(w+d w)$ is

$$
\Omega(w+\delta w)=e^{i(w+\delta w)}=e^{i \int_{0}^{1} d s\left(w_{s}+\delta w_{s}\right)}
$$




$$
\begin{aligned}
& =e^{i \int_{0}^{1} d s w_{s}}+i \int_{0}^{1} e^{i \int_{0}^{1} d s w_{s}} \delta w_{s^{\prime}} d s^{\prime} \\
& =\Omega(w)+i \int_{0}^{1} d s^{\prime}\left[e^{i \int_{s^{\prime}}^{1} d s w_{s}} \delta w_{s^{\prime}} e^{i \int_{0}^{s^{\prime}} d s w_{s}}\right] \\
& =\Omega(w)+i \int_{0}^{1} d s^{\prime}\left[e^{i\left(1-s^{\prime}\right) w_{s^{\prime}}} \delta w_{s^{\prime}} e^{i s^{\prime} w}\right] \\
& =\left(\mathbb{I}+i \int_{0}^{1} d s e^{-i s w} T^{a} e^{i s w} \delta w^{a}\right) \Omega(w) .
\end{aligned}
$$

If we define

$$
\Sigma^{a}(s) \equiv e^{-i s w} T^{a} e^{i s w}
$$

and $\sigma^{a b}(s)$ is such a way that

$$
\Sigma^{a}(s)=\sigma^{a b}(s) T^{b}
$$

then it is obvious that

$$
\dot{\Sigma}^{a}(s)=-i\left[w, \Sigma^{a}(s)\right]
$$

and then

$$
\dot{\sigma}^{a b}=w^{c} f^{c f b} \sigma^{a f} .
$$

If we define $\gamma^{a b} \equiv f^{a b c} w^{c}$ then

$$
\dot{\sigma}=\sigma \gamma \Longrightarrow \sigma=e^{s \gamma}
$$

and then

$$
\Sigma^{a}=\left(e^{\gamma s}\right)^{a b} T^{b} \Longrightarrow \int_{0}^{1} d s \Sigma^{a}=\left[\frac{e^{\gamma}-\mathbb{I}}{\gamma}\right]^{a b} T^{b} .
$$

We can conclude that

$$
\begin{aligned}
\Omega(w+\delta w) & =\left(\mathbb{I}+i \Phi^{a b}(w) T^{b} \delta w^{a}\right) \Omega(w) \\
\Phi^{a b}(w) & \equiv\left[\frac{e^{\gamma}-\mathbb{I}}{\gamma}\right]^{a b} .
\end{aligned}
$$

As $\Omega$ is a unitary matrix

$$
\Omega(w+\delta w) \Omega^{\dagger}(w+\delta w)=\mathbb{I} ;
$$

substituting the (43) in the last equation and considering only linear terms in $\delta w$ we obtain

$$
\left(\Phi^{a b}(w) T^{b} \delta w^{a}\right)^{\dagger}=\Phi^{a b}(w) T^{b} \delta w^{a}
$$


and then

$$
\Omega^{\dagger}(w+\delta w)=\Omega^{\dagger}(w)\left(\mathbb{I}+i \Phi^{a b}(w) T^{b} \delta w^{a}\right) .
$$

If we remember that

$$
\begin{aligned}
A_{\mu}^{\Omega}(x) & =\Omega(x) A_{\mu}(x) \Omega^{\dagger}(x)-\frac{i}{g} \Omega(x) \partial_{\mu} \Omega^{\dagger}(x) \\
\Omega(x) & =e^{i w(x)}
\end{aligned}
$$

it is easy to verify that

$$
\delta F(\Omega)=-\frac{2}{g} \int d^{4} x\left(\partial_{\mu} A_{\mu}^{\Omega}\right)^{a} \Phi^{a b}(w) \delta w^{b}
$$

and then

$$
\frac{\delta F(\Omega)}{\delta w^{b}}=-\frac{2}{g}\left(\partial_{\mu} A_{\mu}^{\Omega}\right)^{a} \Phi^{a b}(w)
$$

\section{References}

[1] P. Bacilieri et al. (Ape collaboration), Nucl. ZPhys. B 317 (1989) 509; Nucl. Phys. B 343 (1990) 228.

[2] L. Maiani, G. Martinelli, C.T. Sachrajda Nucl. Phys. B 368 (1992) 281-292.

[3] G. Martinelli, C. Pittori, C.T. Sachrajda, M. Testa and A. Vladikas, Nucl. Phys. B 445 (1995) 81-108.

[4] J.E. Mandula, M. Ogilvie, Phys. Lett. B 185 (1987) 127.

[5] R. Gupta, G. Guralnik, G. Kilcup, A. Patel, S. R. Sharpe and T. Warnock, Phys. Rev. D 36 (1987) 2813.

[6] C. Bernard, A. Soni and K. Yee, Nucl. Phys. B (Proc. Suppl.) 20 (1991) 410.

[7] P. Marenzoni, G. Martinelli, N. Stella, M. Testa, Phys. Lett. B 318 (1993) 511.

[8] P. Marenzoni, G. Martinelli, N. Stella, Nucl. Phys. B 455 (1995) 339-356.

[9] C. Bernard, C. Parrinello and A. Soni, Nucl. Phys. B (Proc. Suppl.) 30 (1993) 535 and Phys. Rev. D 49 (1994) 1585.

[10] E. Marinari, C. Parrinello, R. Ricci, Nucl. Phys B 362 (1991) 487-497.

[11] Ph. de Forcrand, J.E. Hetrick, A. Nakamura and M. Plewnia, Nucl. Phys. B (Proc. Suppl.) 20 (1991) 194. 
[12] C. Parrinello, S. Petrarca, A. Vladikas, Phys. Lett. B 268 (1991) 236-240.

[13] M.L. Paciello, C. Parrinello, S. Petrarca, B. Taglienti and A. Vladikas, Phys. Lett. B 276 (1992) 163-167, erratum-ibid. B 281 (1992) 417.

[14] V.N. Gribov, Nucl. Phys B 139 (1978) 1-19, For a review of the Gribov ambiguity in the continuum see for example S. Sciuto, Phys. Rep. 49 (1979) 181 and references therein.

[15] K.G. Wilson, Recent developments in gauge theories, Proc. NATO Advance Study Institute (Cargése,1979), eds. G.'t Hooft et al.

[16] J.E. Mandula, M. Ogilvie, Phys. Lett. B 248 (1990) 156-162.

[17] C.T.H. Davies et al, Phys. Rev. D 37 (1988) 1581-1588.

[18] L. Conti, C. Parrinello, S. Petrarca and A. Vladikas, Phys. Lett. B 373 (1996) 164.

[19] C. Itzykson, J.B. Zuber Quantum Field Theory, Mc.Graw-Hill 1980.

J. Zinn-Justin, Quantum Field Theory and Critical Phenomena, Oxford University Press 1989.

[20] N. Cabibbo, E. Marinari, Phys. Lett. B 119 (1982) 387-390.

[21] G.C. Rossi, M. Testa, Nucl. Phys B 163 (1980) 109-132.

[22] R.P. Feynman, Phys. Rev. 84 (1951) 108-128.

[23] G. Martinelli, S. Petrarca, C.T. Sachrajda, A. Vladikas, Phys. Lett. B 311 (1993) 241-248.

[24] M.L. Paciello, S. Petrarca, B. Taglienti and A. Vladikas, Phys. Lett. B 341 (1994) 187-194.

[25] P. de Forcrand, J. E. Hetrick, Nucl. Phys. (Proc. Suppl.) 42 (1995) 861-866. 